\title{
Apuntes para el estudio de la transhumancia en el valle de Camarones (provincia de Tarapacá, Chile)
}

Virgilio SchiapPacasse F.* y Hans Niemeyer F.*

Al describir en 1963 los yacimientos preagrícolas de Conanoxa, en la quebrada de Camarones, interpretamos dicho sitio como campamentos transitorios pertenecientes a grupos recolectores cazadores transhumantes (Niemeyer y Schiappacasse 1963). ${ }^{1}$

En la actualidad, casi 10 años después, se ha acrecentado el interés de los arqueólogos chilenos por el estudio de la adaptación humana en las regiones áridas y semiáridas del país y por el papel que le pudo haber correspondido a la transhumancia en este proceso de adaptación, además de su probable contribución en la domesticación de plantas y animales. Se explica entonces que haya sido escogido este tema para uno de los Simposios del Primer Congreso del Hombre Andino.

Con la perspectiva que aporta el paso del tiempo y un mayor conocimiento arqueológico de la quebrada de Camarones cabría preguntarse si todavía consideramos válidos los conceptos formulados en 1963.

Para responder a esta pregunta deberíamos precisar previamente qué entendemos por transhumancia y analizar en seguida los elementos de juicio que disponemos de los sitios en discusión, a los cuales nos referiremos de aquí en adelante como "Cultura de Conanoxa".

En geografía humana se utiliza actualmente el término de transhumancia para definir el desplazamiento estacional del ganado doméstico. Su origen se debería, a decir de Forde (1966):

\footnotetext{
Sociedad Chilena de Arqueología.

Los sitios pertenecientes a esta cultura, estudiados más exhaustivamente a la fecha, se encuentran sobre dos terrazas fluviales de la quebrada de Camarones en la localidad de Conanoxa, a unos $40 \mathrm{~km}$ de la costa. La superficie de estos yacimientos arqueológicos, expuesta por la erosión, varía entre $250 \mathrm{a} 1250 \mathrm{~m}^{2}$ y el espesor de las basuras, no mayor de $0.50 \mathrm{~m}$ de profundidad, consiste en varios pisos de ocupación superpuestos indicando residencias temporales repetidas.
}

“Esta migración estacional de los cuarteles de invierno a los de verano, en beneficio del ganado, practicada por los pueblos más adelantados que habitan en zonas semiáridas y montañosas [se refiere a los habitantes de Persia Central], estaba antiguamente muy difundida en el sur de Europa y se la denomina transhumancia, precisamente por el término usado en España, en donde hasta hace poco tiempo estaba excepcionalmente bien desarrollada".

Nosotros nos limitaremos en esta oportunidad solamente a la transhumancia practicada durante el estadio de caza-recolección, como sinónimo de una forma de seminomadismo estacional, sin tomar en consideración la transhumancia de tiempos posteriores dentro de un sistema de economía "mixta" agrícola-ganadera, de la cual hay evidencias arqueológicas en la quebrada de Camarones durante el Período Tardío (Niemeyer, et al. 1971).

En el análisis de los patrones de residencia pueden reconocerse, entre los extremos de una residencia sedentaria y de una nómade estricta, varias formas intermedias (a las cuales se ha aplicado el nombre genérico de seminomadismo), atendiendo al carácter permanente o semipermanente de los campamentos base, a la ubicación de los campamentos transitorios y a la amplitud de los desplazamientos entre éstos (ver esquema).

La transhumancia es una forma de seminomadismo que lleva involucrada una relación directa con el cambio estacional del ambiente. La arqueóloga americana Emma L. Davis (cuyas ideas han influenciado en forma importante nuestro pensamiento) ha definido esta "transhumancia estacional" como "la práctica de ciertos grupos humanos de cambiar de residencia en forma regular y tradicional como respuesta al cambio estacional en la disponibilidad de los recursos naturales" (Davis 1963).

Las condiciones fundamentales del medio ambiente que determinaría este proceso de adaptación serían: 
Patrones de residencia

\begin{tabular}{|c|c|c|c|c|c|}
\hline \multirow{4}{*}{ Movilidad creciente } & \multicolumn{2}{|c|}{ Invierno } & \multicolumn{2}{|c|}{ Verano } & Residencia \\
\hline & \multicolumn{4}{|c|}{ Residencia permanente } & Sedentarios estrictos \\
\hline & $\begin{array}{l}\text { Campamentos } \\
\text { base }\end{array}$ & $\begin{array}{l}\text { Permanentes o } \\
\text { semipermanentes }\end{array}$ & $\begin{array}{l}\text { Campamentos } \\
\text { transitorios }\end{array}$ & $\begin{array}{l}\text { Variables o } \\
\text { definidos }\end{array}$ & $\begin{array}{l}\text { Seminómadas o } \\
\text { semisedentarios }\end{array}$ \\
\hline & \multicolumn{4}{|c|}{ Residencia transitoria } & Nómadas estrictos \\
\hline
\end{tabular}

a) Un hábitat árido con disponibilidad limitada de recursos.

b) Presencia de varios microambientes, los cuales aportan sus recursos en diferentes épocas del año. Estos microambientes se ubican a diferente altitud en el modelo de Davis, y

c) Grandes fluctuaciones estacionales de la temperatura que regulan la permanencia del grupo humano en un determinado ambiente durante el ciclo anual.

Para el reconocimiento arqueológico de una transhumancia como la propuesta es importante tener en consideración los siguientes hechos:

1) En el cambio de residencia anual el grupo humano puede agruparse y en otras ocasiones dispersarse, dando lugar a sitios de diferentes magnitudes, que en ocasiones son difíciles de individualizar, sea por la dispersión en superficie o bien por la ausencia o escasez de artefactos diagnósticos.

2) Como los diferentes microambientes disponen de diferentes recursos, los utensilios empleados en su explotación variarán en concordancia, dando lugar a restos arqueológicos que pueden simular a primera vista "diferentes culturas".

3) El hallazgo de restos orgánicos pertenecientes a otro microambiente en las basuras de un sitio no debe ser considerado, en ausencia de otras evidencias, como demostrativo de transhumancia, pudiendo estar involucrados otros mecanismos causales, sean éstos naturales o culturales (el trueque, por ejemplo).

Es evidente que la práctica de la transhumancia puede enfrentar al arqueólogo con problemas difíciles de interpretar correctamente sin el auxilio de otros elementos de juicio, como pueden ser los antecedentes etnohistóricos.
Analizaremos ahora el caso particular de la "Cultura de Conanoxa".

En el curso de la quebrada de Camarones, desde la desembocadura hasta sus cabeceras de precordillera y el altiplano que las alimenta hacia el oriente, pueden reconocerse cinco zonas ecológicas que van escalonándose desde el nivel del mar hasta los $4500 \mathrm{~m}$ de altura.

1) Litoral desértico.

2) Oasis de valle.

3) Estepas arbustivas.

4) Tolar de puna.

5) Puna.

Al presente hemos individualizado sitios pertenecientes a la "Cultura de Conanoxa" solamente en las zonas 1 y 2 y no disponemos de evidencias directas o indirectas que demuestren la explotación por esta cultura de las zonas ecológicas restantes. ${ }^{2}$

2 Consideramos como sitios pertenecientes a esta cultura, además de los yacimientos de Conanoxa ya nombrados, los estudiados por nosotros en las terrazas de Cuya y de Chaca, todos ellos comprendidos en la zona ecológica 2 de oasis de valle. En la zona litoral comprendemos los sitios que ubicamos en el extremo oriental de la terraza sur de la desembocadura de la quebrada, y provisoriamente al sitio en el margen opuesto de la desembocadura, en vías de investigación por Dauelsberg y colaboradores, dado a conocer en forma preliminar en el VI Congreso de Arqueología Chilena en 1971. La punta de proyectil, a la cual se hizo referencia, es uno de los elementos característicos que se repite en todos estos sitios, por lo que creemos puede ser considerada como un "fósil guía" de utilidad para identificar futuros yacimientos de esta cultura. El hallazgo reciente que hiciéramos de algunas puntas de proyectiles a orillas del Salar de Surire que pudieran atribuirse a la "Cultura de Conaxona" proporciona una evidencia insuficiente por ahora. Es oportuno destacar que mientras en las tierras bajas la existencia de quebradas separadas entre sí por extensas pampas desérticas es un factor de diversificación cultural, la puna es, por el contrario, un factor unificador. Nos ha llamado la atención, por ejemplo, la semejanza tipológica 
Sin embargo, estas evidencias negativas no deben ser consideradas como pruebas concluyentes debido a la posibilidad que algunos campamentos transitorios pueden haber pasado inadvertidos a nuestra exploración o hayan sido destruidos o enmascarados por la actividad humana posterior o por causas naturales (aluviones, por ejemplo).

Las diferentes zonas ecológicas de la quebrada de Camarones están comprendidas dentro de un bioma general desértico, pero no se dispone de datos científicos referentes a variaciones relativas en el ciclo biológico de plantas y animales entre estas zonas. Tampoco los cambios estacionales de temperatura son de magnitudes tales como para impedir la permanencia de grupos humanos en determinadas épocas del año en algunas de estas zonas.

En los sitios atribuidos a la "Cultura de Conanoxa" se han documentado, ya sea por el análisis de sus basuras como por los restos de utensilios empleados, las siguientes actividades de subsistencia:

En la zona 2 de "oasis de valle": a) recolección de productos vegetales; b) pesca o recolección del camarón de río; c) caza de pequeños y grandes mamíferos terrestres y de aves (p. e., vizcacha y guanaco); d) no debe desestimarse la probable recolección de insectos, reptiles y batracios.

En la zona 1 o litoral, a las actividades enumeradas más arriba se agregan las siguientes actividades específicas para esta zona: e) pesca marítima desde la costa; f) recolección de moluscos; g) caza de mamíferos acuáticos (lobo de mar).

Todas estas actividades pudieron haber sido ejecutadas desde campamentos ubicados en el litoral y complementadas con expediciones realizadas dentro del marco de una jornada sin necesidad de campamentos transitorios adicionales.

Sin embargo, deben considerarse algunos factores que pudieran haber condicionado un padrón de residencia transhumante:

1) Limitación numérica de los recursos del valle en la zona litoral con la necesidad consiguiente de realizar expediciones de mayor aliento

del material lítico del Salar de Surire con los materiales de los salares más meridionales. hacia el valle interior que implicaran varias jornadas.

2) La vizcacha tiene como hábitat natural los ambientes rocosos y pedregosos. Las angosturas de Conanoxa y de Taltape, situadas al interior, ofrecen condiciones ideales al respecto, no comparables con ninguna existente en el litoral.

3) La biología del camarón de río condiciona una migración de los ejemplares hembras fecundados hacia la desembocadura durante el período de crecida de la quebrada (período de las lluvias estivales). Al bajar el caudal del río durante la estación invernal, las hembras y larvas emprenden una migración inversa aguas arriba.

Por su abundancia en las basuras, el camarón de río debió constituir un componente importante de la dieta y es posible, entonces, que estos grupos humanos tuvieran que desplazarse por el curso de la quebrada siguiendo la disponibilidad del camarón.

4) La explotación de los nódulos de calcedonia como materia prima es otro probable factor determinante del establecimiento de campamentos en Conanoxa, pero desde luego sin ningún determinismo estacional. Precisamente en la entrada oriente de la Angostura de Conanoxa se produce con evidente discordancia angular el contacto entre la formación terciaria, algunos de cuyos estratos están formados por calcedonia, y la formación mesozoica que persiste de allí hasta el mar, estando ausente la primera en el tramo de los últimos $40 \mathrm{~km}$. Corrobora lo anterior la escasa frecuencia de desechos de talla de calcedonia que se encuentran en los sitios del litoral.

5) El hallazgo de espinas de cactos en las basuras de los yacimientos en la costa determinaría un límite mínimo del desplazamiento hacia el interior del valle abarcando la tercera zona ecológica de estepas arbustivas.

De esta breve reseña puede concluirse que la demostración de una actividad transhumante, en base exclusivamente a evidencias arqueológicas, puede tropezar con dificultades insuperables.

En el ejemplo específico de la "Cultura de Conanoxa" su carácter transhumante debe considerarse por ahora 
como una hipótesis de trabajo, siendo necesario un mayor acopio de información antes de poder considerarlo como un hecho científicamente demostrado.

El acervo material de esta cultura se caracteriza, además de utensilios elaborados de fibras vegetales y de fanéreos y cueros de animales, por una variada gama de litos tallados en calcedonia, entre los cuales destaca una punta de proyectil ovalada de tamaño pequeño a mediano (tipo III de nuestra clasificación). Cepillos o tajadores elaborados de guijarros y morteros de piedra completan este inventario material.

Actualmente disponemos de tres fechados de $\mathrm{C}^{14}$ para el sitio Cxa W (a) (Schiappacasse y Niemeyer 1969):
1) Muestra IVIC $-175=3740 \pm 130$ años de edad

2) Muestra IVIC $-875=4020 \pm 110$ años de edad

3) Muestra IVIC $-876=3970 \pm 120$ años de edad

IVIC: Instituto Venezolano de Investigaciones.

Frente al criterio que favorece la práctica de una transhumancia "transversal", desde la costa hasta la puna, en el estudio de "caza-recolección" pudiera oponerse como hipótesis de trabajo alternativa la existencia de dos tradiciones transhumantes: una propia de las "tierras bajas" siguiendo el curso de las quebradas, y otra de las "tierras altas" que explotara los diversos microambientes de la puna y valles cordilleranos.

\section{REFERENCIAS CITADAS}

DAVIS, E. L., 1963. The Desert Culture of the Western Great Basin: A lifeway of seasonal transhumance. American Antiquity 29 (2): 202.

FORDE, C. D., 1966. Habitat, economy and society. Versión española de la $15^{\text {a }}$ edición inglesa. Industrias Gráficas García, Barcelona.

NIEMEYER, H. y V. SCHIAPPACASSE, 1963. Investigaciones arqueológicas en las terrazas de Conanoxa, valle de Camarones (provincia de Tarapacá). Revista Universitaria, año XLVIII. Anales de la Academia Chilena de Ciencias Naturales 26.
NIEMEYER, H., V. SCHIAPPACASSE e I. SOLIMANO, 1972. Padrones de poblamiento en la quebrada de Camarones, prov. de Tarapacá (estudio preliminar que comprende el sector medio y superior del valle). Actas del VI Congreso de Arqueología Chilena. Boletín de Prehistoria, $\mathrm{n}^{\circ}$ especial.

SCHIAPPACASSE, V. y H. NIEMEYER, 1969. Comentario a tres fechas rediocarbónicas de sitios arqueológicos de Conanoxa (valle de Camarones, provincia de Tarapacá). Noticiario Mensual del Museo Nacional de Historia Natural, XIII (151). 